\title{
Hermansky-Pudlak Syndrome 1
}

National Cancer Institute

\section{Source}

National Cancer Institute. Hermansky-Pudlak Syndrome 1. NCI Thesaurus. Code C150367.

An autosomal recessive sub-type of Hermansky-Pudlak syndrome caused by mutation(s) in the HPS1 gene, encoding Hermansky-Pudlak syndrome 1 protein. This sub-type is associated with pulmonary fibrosis. 